\title{
PERFORMANCE ANALYSIS OF ECONOMIC MODEL AND RADIO RESOURCE MANAGEMENT IN HETEROGENEOUS WIRELESS NETWORKS
}

\author{
Nilkantha Chakraborty \\ Cognizant Technology Solution India Pvt. Ltd \\ Mepz Economic Zone, Chennai, Tamil Nadu, India \\ nil.0308@yahoo.in
}

\begin{abstract}
Radio resource management (RRM) is the system level control of co-channel interference and other radio transmission characteristics in wireless communication systems. In this paper we first analyze a utility function based access selection (UFAS) method. Then we investigate a radio resource management and network selection scheme in heterogeneous wireless network. We propose an economic model to allocate radio resources for Code Division Multiple Access (CDMA) Networks and Wireless local Area (WLAN) networks. Moreover we formulate and solve the optimization problem in WLAN and CDMA networks and simulate those equations using MATLAB. Simulated results show that the proposed scheme can achieve more number of users and more social welfare which outperform the utility function based access selection method.
\end{abstract}

\section{KEYWORDS}

Radio resource management (RRM), UFAS, proposed economic model, maximum network welfare.

\section{INTRODUCTION}

The ever growing demand for multimedia services, high mobility and global connectivity has resulted in recent years in an exploration of new technologies for wireless communication systems. Interworking between 3G mobile system and WLAN system are gaining high interest. As a matter of fact, 3GPP has organized task forces to investigate the interworking between WLAN and CDMA networks. Quality of service (QOS) can provide the means to manage the network resources. QoS allows for better use of the existing network infrastructure, improve service to the network users and reduces the cost of providing these services. One of the main goals of QoS is to provide priority including dedicated bandwidth, controlled jitter and latency and improved loss characteristics. A WLAN provides wireless network communication over short distances using radio or infrared signals instead of traditional network cabling. Network security remains an important issue for WLANs. WLANs are built by attaching a device called the access point (AP) to the edge of the wired network. WLAN system can be a complementary radio-access technology to $3 \mathrm{G}$ systems in providing more bandwidth and economic revenues. Mobile nodes in such heterogeneous wireless networks are expected to have the capability of selecting a proper access network to ensure service consistency and continuity. Radio resource management (RRM) 
involves strategies and algorithms for controlling parameters such as transmit power, channel allocation, data rate, error coding scheme, modulation scheme, handover criteria etc. One of the objective is to use the limited radio spectrum resources and radio network infrastructure as efficiently as possible. Heterogeneous network is basically used in wireless network using different access technologies. A wireless network which provides services through wireless LAN and is able to maintain services when switching to a cellular network is called heterogeneous networks. We have simulated for Utility function based access selection method (UFAS). This method has been proposed to determine which cell is most suitable for a mobile node's service request in heterogeneous WCDMA and WLAN networks. UFAS method achieves larger system throughput than a Policy - Enabled (PE) handoff decision method. This method can reduce handoff occurrence rate.

\section{INTEGRATION OF WLAN AND UMTS:}

In this paper, we consider the heterogeneous networking environment that is built on CDMA and WLAN wireless-access technologies. The internetworking architecture can either be a tightly coupled case or a loosely coupled case, as shown in Figure 1.

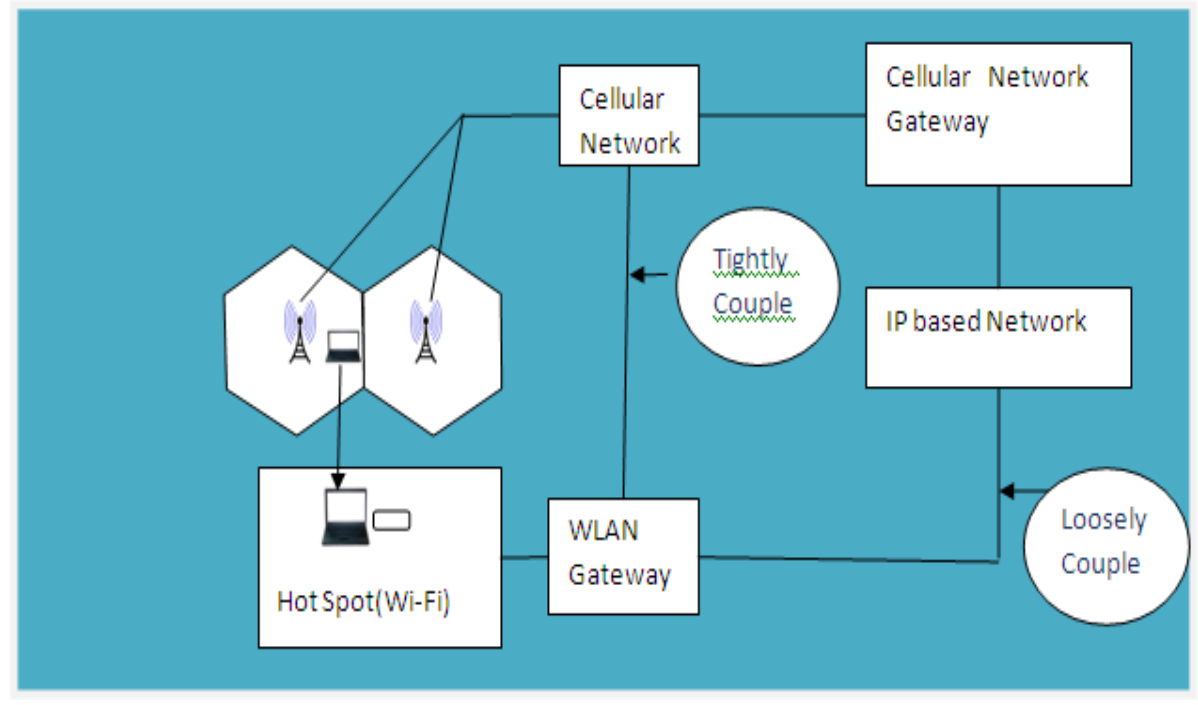

Figure 1. Internetworking architecture between WLAN and cellular network

\section{Loose Coupling:}

In this approach, the WLAN requires no direct links to the $3 \mathrm{G}$ cellular network, and less control overhead. The gateway directly connects the WLANs to the internet backbone. The main advantage of the loose coupling approach is the independent deployment of the two domains is needed for internetworking i.e. less interdependency. In this implements mobile IP, authorization and accounting service to integrate with the UMTS home AAA server. This server enables exchanging accounting information and billing information between WLAN and UMTS networks. In this architecture, WLAN and cellular networks are two separate access networks. The wireless access network is attached to the internet backbone, and the UMTS network is attached to the cellular (UMTS) core network. The overall architecture is loosely- coupled because the data path of the WLAN is completely separated from the data path of the UMTS network. This integration architecture facilitates billing and authentication but does not provide 
Radio Resource Management (RRM) or QoS support. To overcome this drawback, various mechanism and model have been designed for WLAN and UMTS network.

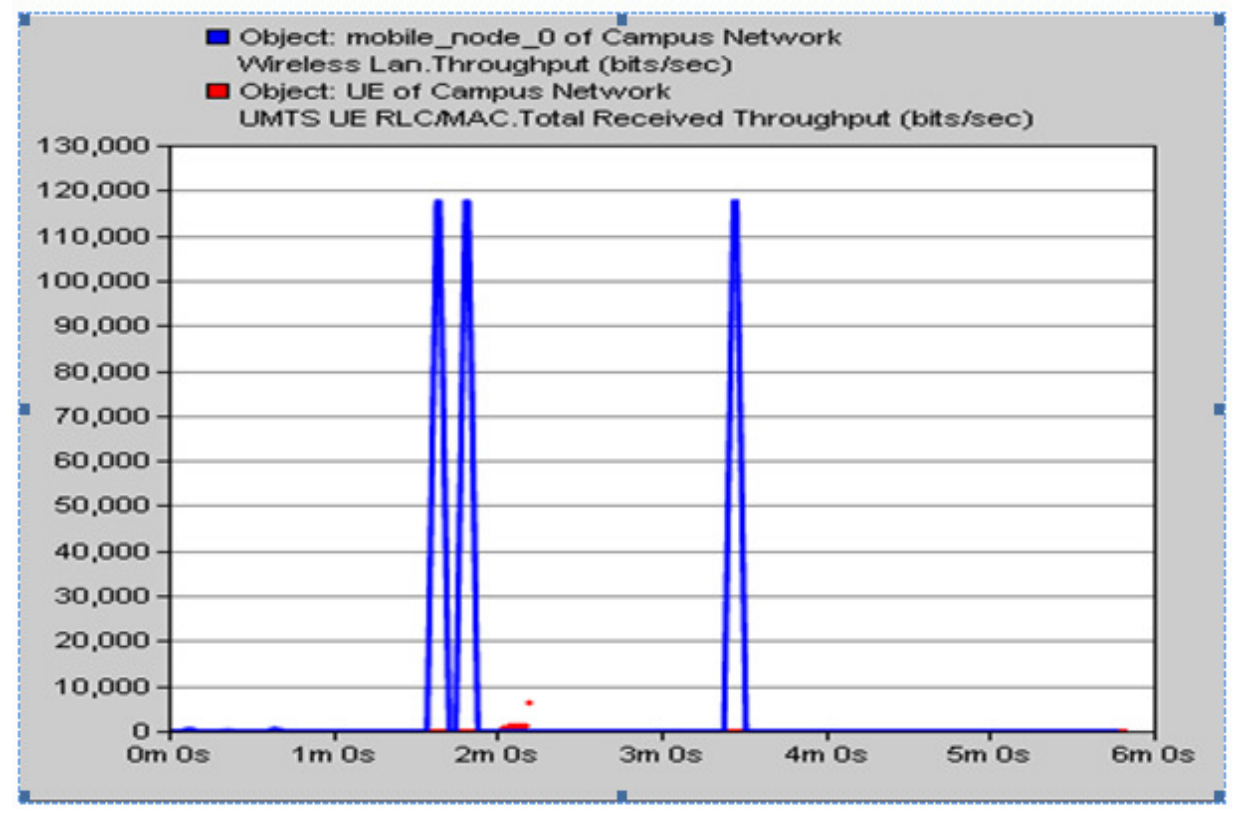

Figure 2. Performance of integrated Network based on throughput using loose coupling

\subsection{Tight Coupling:}

In this approach, the access point (AP) of the WLAN is directly connected to the $3 \mathrm{G}$ cellular core network, and these two networks have to share the entities of authentication, mobility management, and radio-resource allocation. The main advantage of the tight coupling mechanism is the efficient mobility management; i.e., UMTS mobility management features are applied to the integrated networks [11]. It offers service continuity, which includes billing and AAA services. Therefore, inter-domain seamless mobility can be achieved, while reducing handoff latency. The gateway of the WLAN hides the detail of the WLAN network to the UMTS core network and executes all the UMTS protocols (mobility management, authentication, authorization and accounting) requires in a UMTS radio access network (RAN). The different network will share the same authentication, signaling, transport and billing infrastructures independently from protocols used at the physical layer on the radio interface. This approach however has many drawbacks because the UMTS core network interfaces are directly exposed to the WLAN networks. This means that the same operator must own the two networks. The disadvantage of the tight coupling approach is that, as the data traffic of WLAN traverse via the UMTS network, they potentially create a bottle neck in the UMTS network. Moreover, this technique is considered more complicated than the loose coupling technique, because different wireless network protocol stack need to be compatible for such type of integration [12]. 


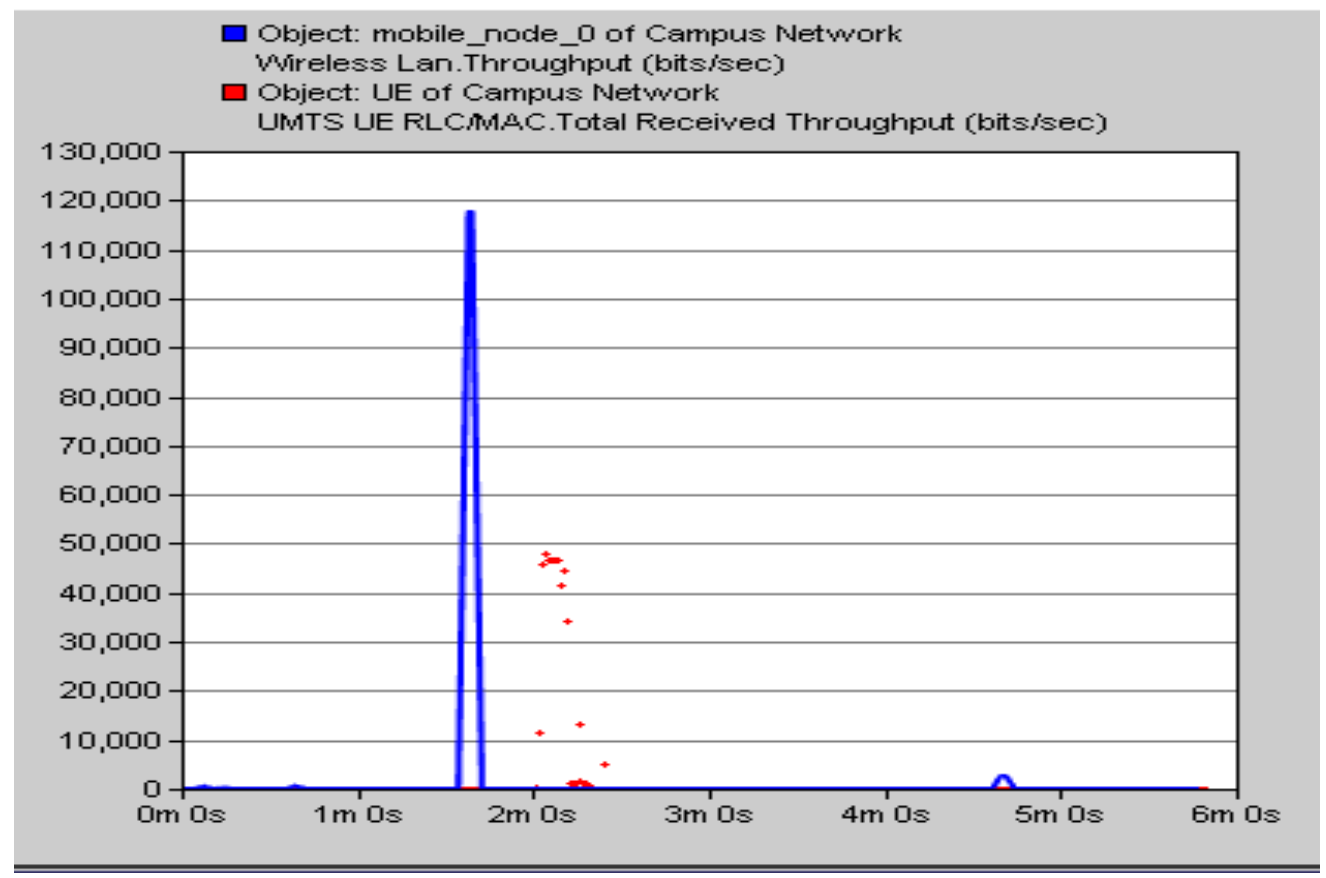

Figure 3. Performance of integrated Network based on throughput using tight coupling

\section{SYSTEM MODEL}

\subsection{New Proposed Economic Model for Radio Resource}

To evaluate network welfare of the allocated bandwidth in heterogeneous wireless network, we have proposed a new economic model with a simulated comparison of Siris and Chens model. Utility function has the characteristic of increasing as well as strictly concave and twice differentiable. Suppose $\mathrm{x}$ is the bandwidth assigned to user and $\mathrm{p}$ is the price per unit bandwidth which is paid by network service provider. If $U$ (b) is the network utility that is attained from bandwidth $b$, the function $U$ (b) - b. p can be used to describe the welfare of a single user from assigned radio resource. If we increase the bandwidth, the user gains more network utility but there is a problem of optional trade off. The welfare model defined by Chen et al. [4] is given as

$$
\begin{gathered}
\mathrm{U}(\mathrm{b}, \mathrm{x})=\exp \left(-(\mathrm{b}-\mathrm{x})^{2}\right) \\
=0, \text { if } \mathrm{b}<\mathrm{x}
\end{gathered}
$$

Where $\mathrm{x}$ is the desired resource mobile user and $\mathrm{b}$ is actual data rate.

The welfare model defined by siris is given as

$$
\mathrm{U}(\mathrm{x})=1-\mathrm{e}^{-\mathrm{b} x}
$$

Our proposed new welfare model can be written as

$$
\left.\mathrm{U}_{\mathrm{p}}(\mathrm{b}, \mathrm{x})=\exp \left(-6(\mathrm{~b}-\mathrm{x})^{2}\right) / \mathrm{x}^{2}\right)
$$

It is normally seen that new call will be dropped if there is not enough bandwidth available to meet the new call. But proposed welfare model contributes a new idea to resolve the issue. 
Simulated graph for three models i.e. Figure 4 is the example. Figure 4 depicts the comparison of three different economic model based on Network Welfare.

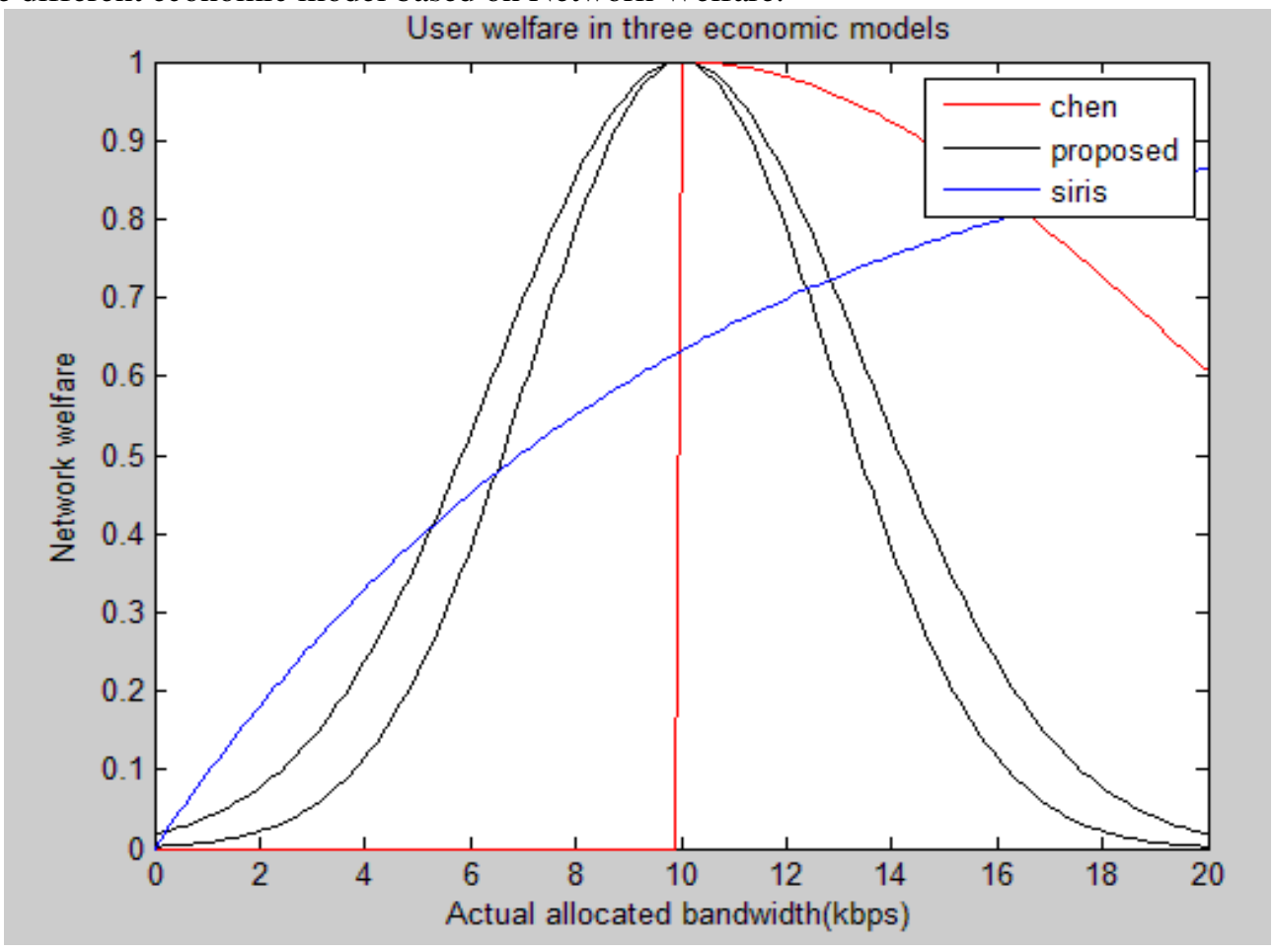

Figure 4. Performance of various economic model using allocated bandwidth

The proposed welfare model has the following features.

- If the actual bandwidth exceeds the user request, the user welfare decreases at a faster speed than the welfare in the model. So there is no chance for over assignment.

- When the assigned bandwidth exactly meets the bandwidth request, the welfare of the user is the maximum.

\subsection{UFAS Mechanism}

Utility function based access selection method can be classified into three parts. Cell classification, utility function computation, target cell determination. Cell classification is to filter out infeasible cells and depends upon following constraint. I) Signal strength constraint Mobile node measures the signal strength. If the received signal strength exceeds threshold value then the cell will be feasible. II) Cell admission constraint - This constraint is used to check if the admittance of the service request affects the QoS requirements of existing connections. III) RF module constraint - We have designed one WCDMA and one WLAN module for an MN. MN can choose any one from both network.

The utility function of a cell for an MN service can be defined as [5]

$$
\mathrm{U}(\mathrm{s})=\begin{array}{llllllllllllllllll}
\mathrm{f} & \mathrm{R}, & \mathrm{n} & (\mathrm{s}) & * & \mathrm{f} & \mathrm{D}, & \mathrm{n} & (\mathrm{s}) & * \mathrm{f} & \mathrm{E}, & \mathrm{n} & (\mathrm{s}) & * \mathrm{f} & \mathrm{v} & \mathrm{n}
\end{array}
$$

Where $\mathrm{f}_{\mathrm{x}, \mathrm{n}}(\mathrm{s}), \mathrm{x}=\mathrm{R}, \mathrm{D}, \mathrm{E}$, and $\mathrm{V}$ denote evaluation functions for data rate, delay, packet error rate (PER), and mobility respectively. 
The function $\mathrm{f}_{\mathrm{R}, \mathrm{n}}(\mathrm{s})$ is defined by

$$
\begin{array}{rlrl}
\mathrm{f}_{\mathrm{R}, \mathrm{n}}(\mathrm{s}) & =\mathrm{G}\left((1+\alpha) \mathrm{R}_{\mathrm{n}}(\mathrm{s}) ; \mathrm{R}_{*}(\mathrm{~s})\right) & \text { when } \mathrm{R}_{\mathrm{n}}(\mathrm{s})<\mathrm{R} *(\mathrm{~s}) \\
& =0 ; \quad \text { otherwise }
\end{array}
$$

$\mathrm{G}(\cdot)$ is given by

$$
\mathrm{G}(\mathrm{x} ; \mu)=\exp \left[-(\mathrm{x}-\mu)^{2} /\left(2^{*} \mu^{2}\right)\right]
$$

The function $\mathrm{f}_{\mathrm{D}, \mathrm{n}}(\mathrm{s})$ and $\mathrm{f}_{\mathrm{E}, \mathrm{n}}(\mathrm{s})$ is defined by

$$
\begin{aligned}
\mathrm{f}_{\mathrm{D}, \mathrm{n}}(\mathrm{s}) & =\mathrm{G}\left((1+\alpha) \mathrm{D}_{\mathrm{n}}(\mathrm{s}) ; \mathrm{D}_{*}(\mathrm{~s})\right) \quad \text { when } \mathrm{D}_{\mathrm{n}}(\mathrm{s})<\mathrm{D}_{*}(\mathrm{~s}) \\
& =0 ; \quad \text { otherwise } \\
\mathrm{f}_{\mathrm{E}, \mathrm{n}}(\mathrm{s}) & =\mathrm{G}\left((1+\alpha) \mathrm{B}_{\mathrm{n}}(\mathrm{s}) ; \mathrm{B}_{*}(\mathrm{~s})\right) \quad \text { when } \mathrm{B}_{\mathrm{n}}(\mathrm{s})<\mathrm{B} *(\mathrm{~s}) \\
& =0 ; \quad \text { otherwise }
\end{aligned}
$$

\subsection{Radio Resource allocation in CDMA uplink}

Consider the QoS requirement of the best effort traffic, in which mobile users are only concerned with the average throughput of successful data transmissions. Therefore, the effective throughput is given by the product $r_{i} p_{i}\left(\gamma_{i}\right)$ of the transmission rate $r_{i}$ and the probability of successful packet transmission $\mathrm{p}_{\mathrm{i}}\left(\gamma_{\mathrm{i}}\right)$. The welfare from mobile user $i$ can be written [2] as $\mathrm{B}\left(\mathrm{r}_{\mathrm{i}} \mathrm{p}_{\mathrm{i}}\left(\gamma_{\mathrm{i}}\right), \mathrm{R}_{\mathrm{i}} \mathrm{p}_{\mathrm{i}}\left(\varphi_{\mathrm{i}}\right)\right)$ where $R_{i}$ is the requested bandwidth by mobile user $i$ and $\varphi_{i}$ is the target SINR to guarantee the BER/PER QoS requirement.

Obviously, $\mathrm{p}_{\mathrm{i}}\left(\gamma_{\mathrm{i}}\right)$ depends on the received SINR $\gamma_{\mathrm{i}}$

i.e., we have

$$
p_{i}\left(\gamma_{i}\right)=\left(1-\operatorname{BER}\left(\gamma_{i}\right)\right)^{n}
$$

Where $n$ is the length of one packet, and $\operatorname{BER}\left(\gamma_{\mathrm{i}}\right)$ is the BER at $\operatorname{SINR} \gamma_{\mathrm{i}}$.

BER has been given as

$$
\mathrm{BER}=1 / 2\left[1-1 / \sqrt{1+\frac{1}{2 \sigma^{2} \gamma_{\mathrm{i}}}+\frac{2}{3 \mathrm{~L}}\left[\left(1+\frac{\mathrm{N}}{5}\right) \mathrm{MK}-1\right]}\right]
$$

Where $L$ means the process gain, $N$ is the number of interference cells, $M$ is the number of multipaths, $K$ is the number of active users, and $\sigma^{2}$ is the power of the Rayleigh envelope. Proposed economic model for CDMA Uplink can be formulated as

$$
\text { Maximize } \begin{aligned}
S_{c}\left(r_{i}, \gamma_{i} \mid \forall_{i}\right) & =\sum_{i=1}^{N_{a}} B_{i}\left(r_{i} p_{i}\left(\gamma_{i}\right), R_{i} p_{i}\left(\emptyset_{i}\right)\right) \\
r_{i} & \geq 0 \quad \gamma_{i} \geq \emptyset_{i} \quad \sum_{i} r_{i} \gamma_{i} \leq \frac{W}{\alpha}
\end{aligned}
$$


From, we know that the maximum network welfare can be achieved only when perfect power control makes the SINR $\gamma_{i}$ equal to $\varphi_{i}$ to provide the BER QoS guarantee for mobile user $i$.

The Lagrangian multiplier method can be applied to obtain the unique solution with the constraint given by

$$
\sum_{i} r_{i} \emptyset_{i}=\frac{W}{\alpha}
$$

Because of the monotonicity of the proposed economic model with respect to the actual assigned bandwidth. Appling Lagrangian formula we have the relationship between all parameters. Equation can be written as

$$
\mathrm{n}=4 \sigma^{2} \gamma_{\mathrm{i}}\left\{\left(\sqrt{1+\frac{1}{2 \sigma^{2} \gamma_{\mathrm{i}}}+\frac{2}{3 \mathrm{~L}}\left[\left(1+\frac{\mathrm{N}}{5}\right) \mathrm{MK}-1\right]}\right)^{3}+1+\frac{1}{2 \sigma^{2} \gamma_{\mathrm{i}}}+\frac{2}{3 \mathrm{~L}}\left[\left(1+\frac{\mathrm{N}}{5}\right) \mathrm{MK}-1\right]\right\}
$$

\subsection{Radio Resource allocation and network welfare in a WLAN system}

Similar to the CDMA network, we analyze in detail the resource-usage constraint in the WLAN, which corresponds to the maximum throughput under the optimum collision probability.

The channel utilization has been formulated as [6]:

$$
\mathrm{R}_{\mathrm{s}}(\mathrm{p})=\frac{\mathrm{p}_{\mathrm{s}}(\mathrm{p}) \mathrm{T}_{\mathrm{s}}}{\mathrm{p}_{\mathrm{i}}(\mathrm{p}) \sigma_{\mathrm{s}}+\mathrm{p}_{\mathrm{s}}(\mathrm{p}) \mathrm{T}_{\mathrm{s}}+\mathrm{p}_{\mathrm{c}}(\mathrm{p}) \mathrm{T}_{\mathrm{c}}}
$$

where $\sigma_{s}$ is the length of a back-off time slot and $T_{s}$ and $T_{c}$ represent the average time period that is associated with one successful transmission and one collision, respectively.

We know,

$$
\begin{aligned}
& \mathrm{T}_{\mathrm{s}}=\mathrm{rts}+\mathrm{cts}+\text { data }+ \text { ack }+3 \mathrm{~s}_{\mathrm{i}} \mathrm{f}_{\mathrm{s}}+\mathrm{d}_{\mathrm{i}} \mathrm{f}_{\mathrm{s}} \\
& \mathrm{T}_{\mathrm{c}}=\mathrm{rts}+\mathrm{cts}+\mathrm{s}_{\mathrm{i}} \mathrm{f}_{\mathrm{s}}+\mathrm{d}_{\mathrm{i}} \mathrm{f}_{\mathrm{s}}
\end{aligned}
$$

Where rts, cts, data, ack, $s_{i} f_{s}$, and $d_{i} f_{s}$ represent the average time of the RTS message, CTS message, data

transmission period, acknowledgement message, short interframe space, and distributed interframe

space, respectively. To optimize the network it can be formulate on the basis of Lagrangian multiplier

method [1].

So it can be formulated as

$$
S_{w}\left(r_{i}, R_{i}\right)=\sum_{i=1}^{m} \exp \left(-\frac{4\left(\omega_{a}-\sum_{i=1}^{m} R_{i}\right)^{2} R_{i}^{2}}{\left(\sum_{i=1}^{m} R_{i}^{2}\right)^{2}}\right)
$$

$\omega_{\mathrm{a}}$ is total available bandwidth in network.

\section{SIMULATION RESULTS AND DISCUSSIONS}

\subsection{Simulation Assumptions and Parameters}

Table 1. Parameters based on CDMA Technology

\begin{tabular}{|l|l|}
\hline Process gain $(\mathrm{L})$ & 1023 \\
\hline Number of multipath(N) & 4 \\
\hline Chip rate(w) & 3.84 \\
\hline
\end{tabular}


International Journal of Computer Networks \& Communications (IJCNC) Vol.5, No.5, September 2013

\begin{tabular}{|l|l|}
\hline Scaling factor & .5 \\
\hline The power of Rayleigh envelop & 2 \\
\hline
\end{tabular}

Table 2. Parameters based on UFAS Mechanism

\begin{tabular}{|l|l|}
\hline Required Data rate (bps) & 2000 \\
\hline Measurement value of Data rate(bps) & 5000 \\
\hline Required delay(msec) & 300 \\
\hline Measurement value of delay(msec) & 100 \\
\hline Required PER & .05 \\
\hline Measurement value of PER & .02 \\
\hline Radius of cell & 300 \\
\hline Number of cell & 19 \\
\hline
\end{tabular}

Table 3. Parameters based on WLAN Technology

\begin{tabular}{|l|l|}
\hline Average time to Request to send(msec) & 20 \\
\hline Average time to Clear to send (msec) & 20 \\
\hline Average time to Data transmission period(msec) & 2 \\
\hline Average time to Acknowledgement(msec) & 15 \\
\hline Number of mobile user & 20 \\
\hline Required bandwidth & 500 \\
\hline
\end{tabular}

\subsection{Evaluation Based on Performance}

Here, we carry out simulated results for both CDMA and WLAN networks. New proposed economic model has been implemented to evaluate the performance. Figure 5 depicts three scenarios as well as two comparisons. Maximum value for Network Social Welfare increases with higher values of Scaling factor and decreases hyperbolically against increasing value of signal quality. Beta remains unchanged at this point of time. On the other scenario, Maximum Network Social Welfare increases with lower values of Beta maintaining SF as unchanged. Figure 6 and 7 are simulated delay and PER performance in UFAS mechanism. UFAS selects the suitable cell according to PER, data rate, delay, and mobility. It is possible

that a cell with mildly lower channel quality (higher PER) has better system throughput or the less number of handoffs. In case of coupled heterogeneous networks we have assumed 12 users at the beginning. Performance of both the maximum network social welfare and the maximum number of users are analyzed here. From Figure 8, the point can be noted that our new proposed mechanism can increase the network social welfare by about $12 \%$ through admitting more users. Single network access mechanism can also improve the network welfare .Figure 9 indicates that our new proposed RRM and access mechanism can have near about $20 \%$ more users than the UFAS mechanism. In case of CDMA network, when the number of mobile users gradually increases, the total social welfare of heterogeneous network is investigated. 
International Journal of Computer Networks \& Communications (IJCNC) Vol.5, No.5, September 2013

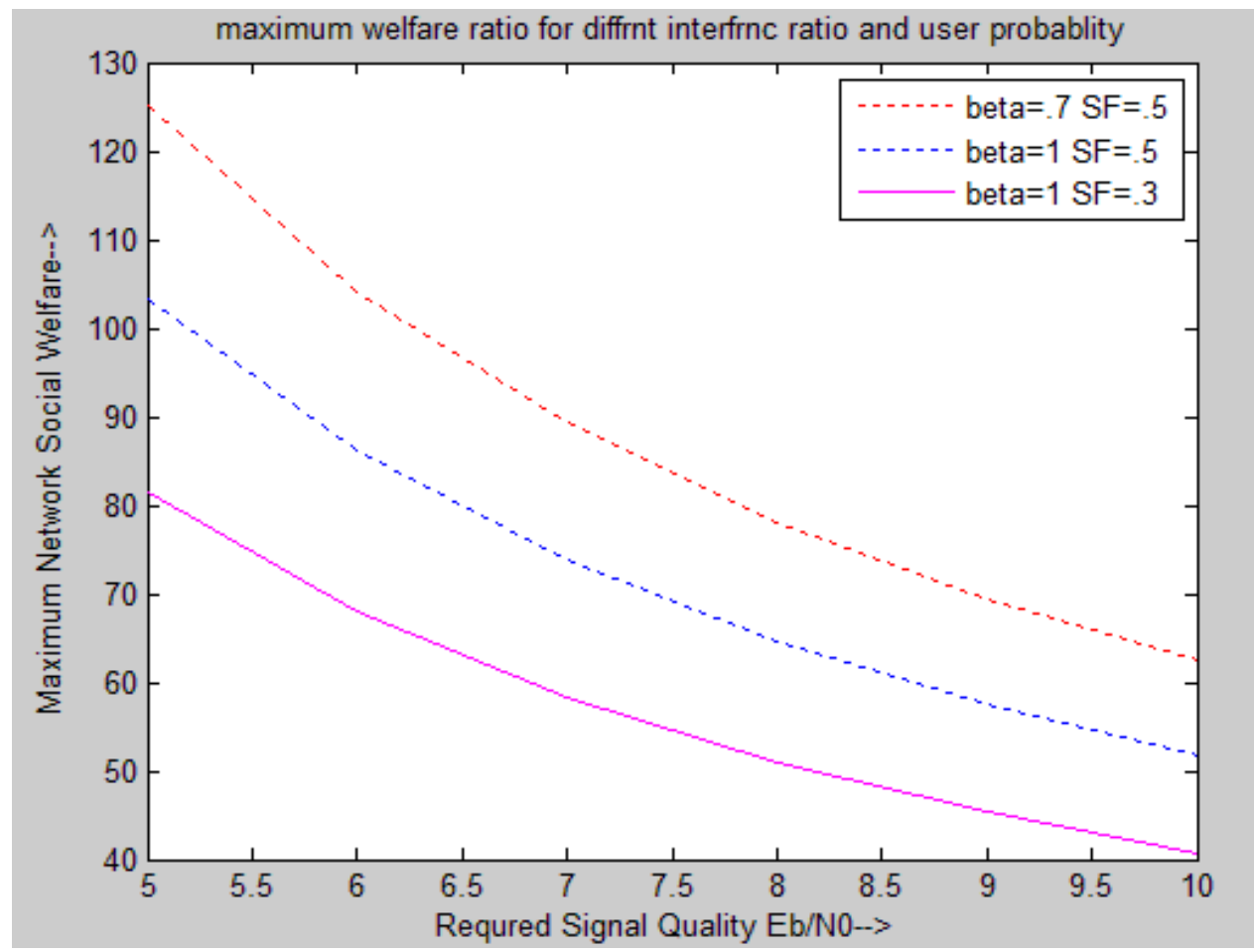

Figure 5. Performance of Maximum welfare ratio using different interference ratio in CDMA

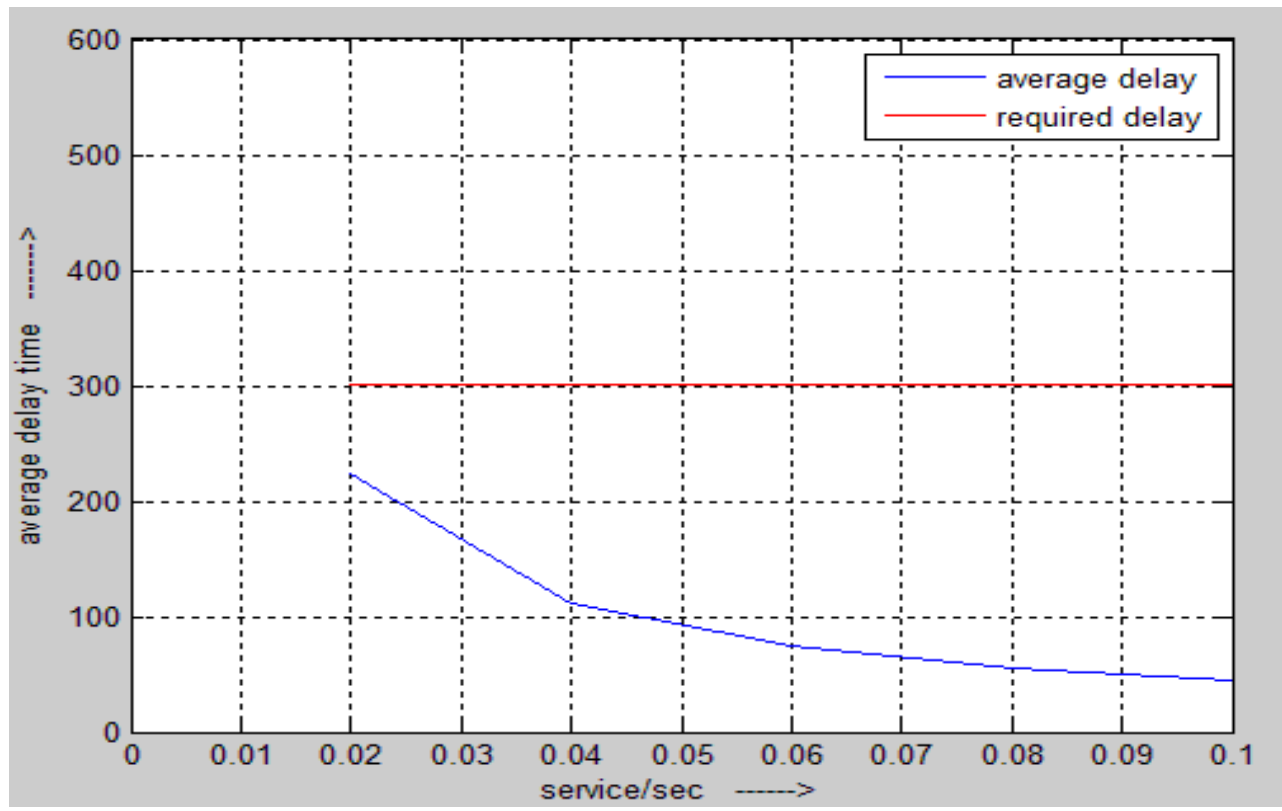

Figure 6. Performance of Delay in UFAS Mechanism 
International Journal of Computer Networks \& Communications (IJCNC) Vol.5, No.5, September 2013

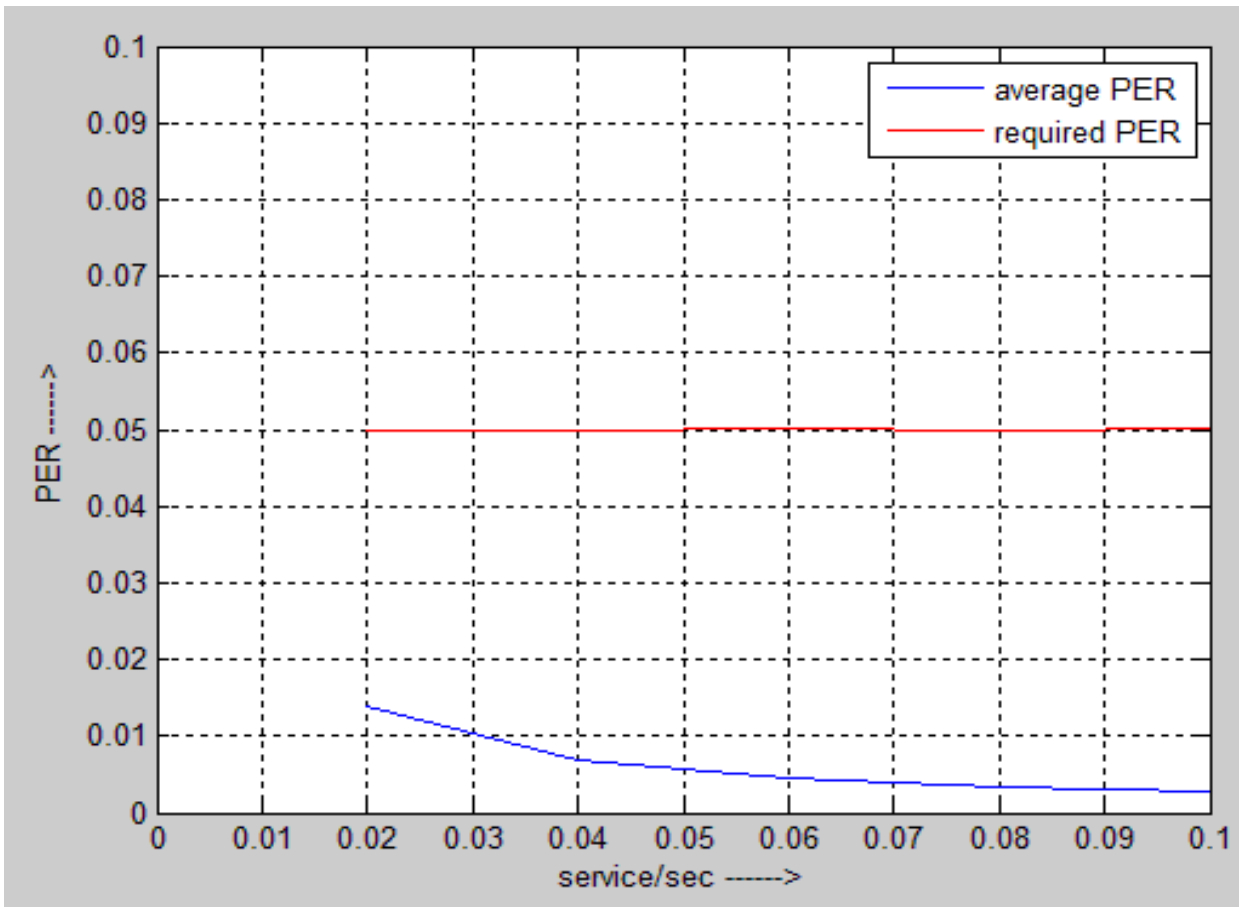

Figure 7. Performance of PER in UFAS Mechanism

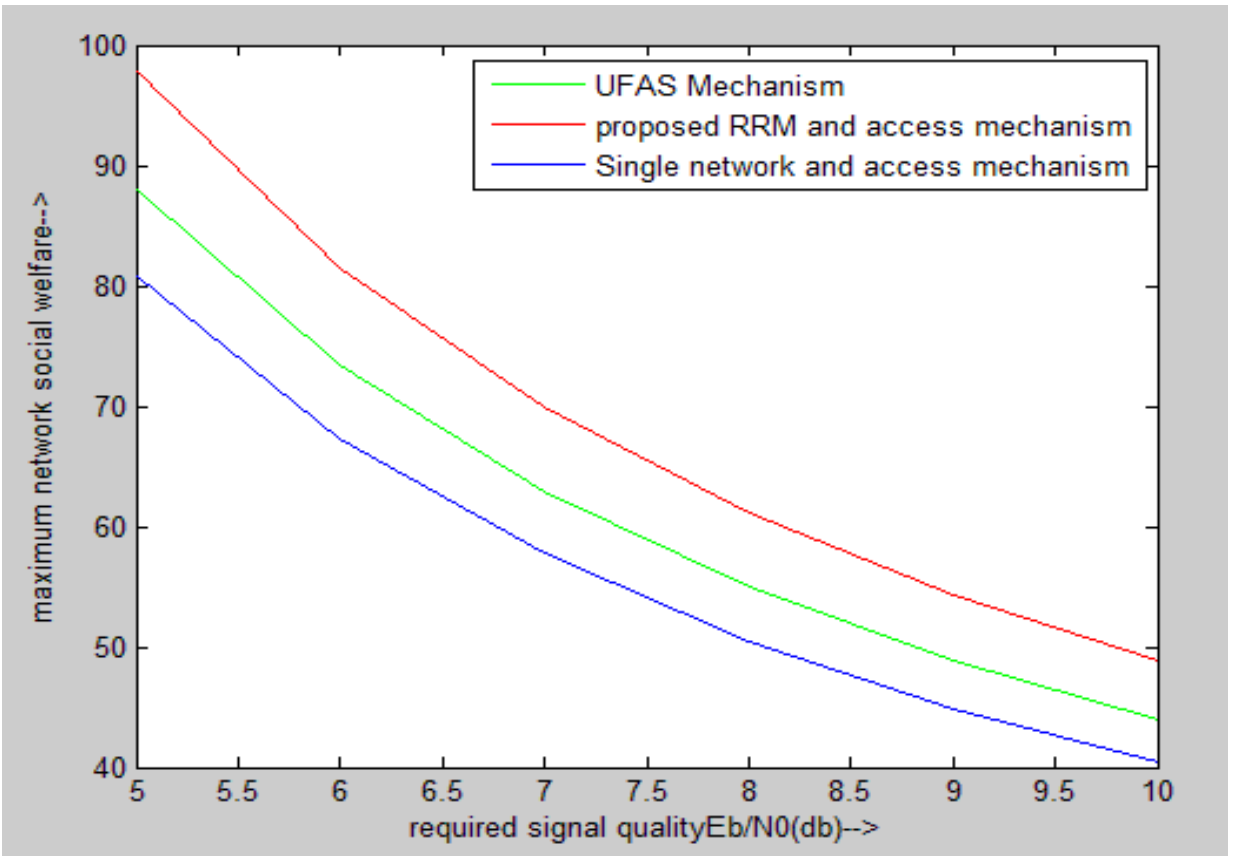

Figure 8. Performance of various mechanism using different guarantee of signal quality 


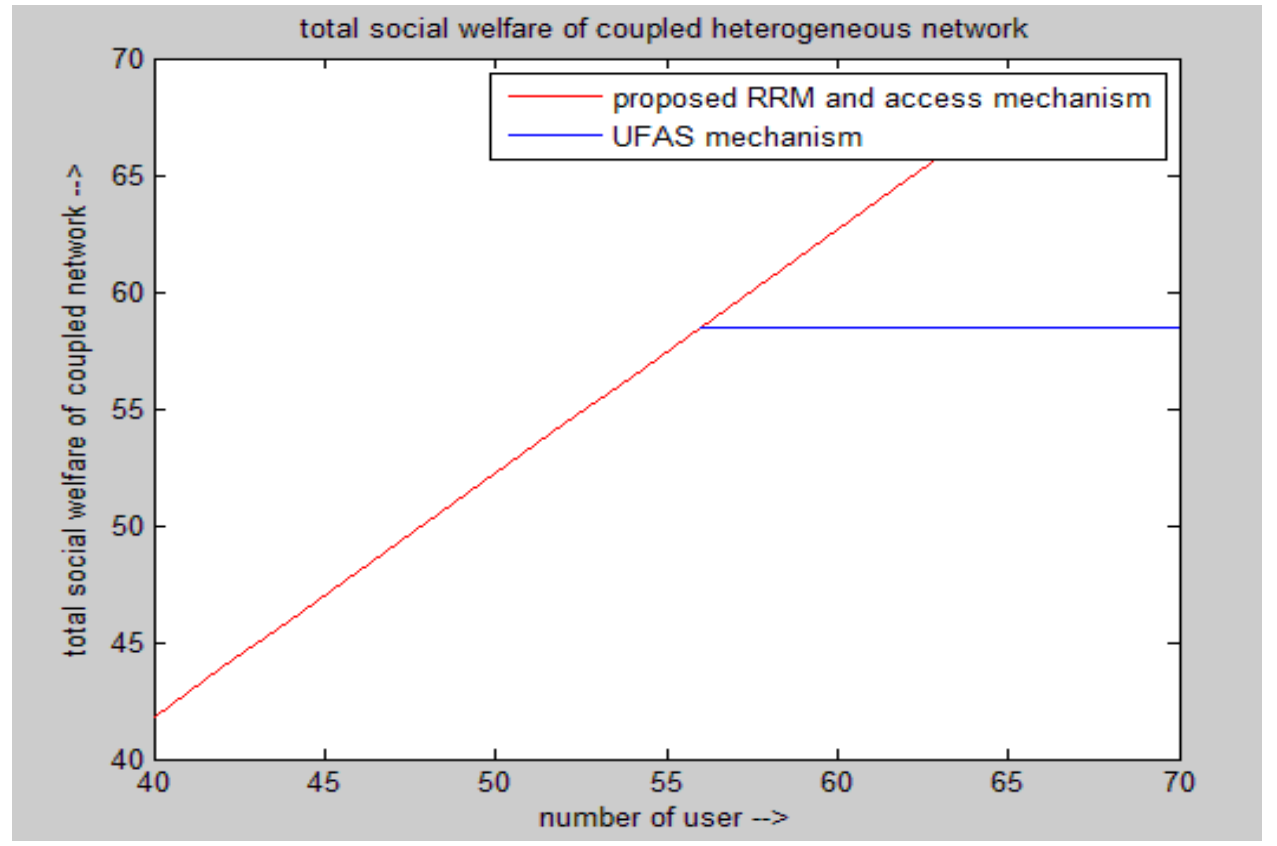

Figure 9. Performance of proposed RRM and UFAS mechanism using total social welfare

\section{CONCLUSIONS}

In this paper, radio-resource usage constraint is allocated to mobile users based on our new proposed economic model. Perfect signal quality is required to maximize the network social welfare. Expression for network welfare based on CDMA and WLAN has been mentioned above. Simulated results for heterogeneous network have concluded that the proposed RRM mechanism achieves maximum network welfare and provides better services compared with other schemes.

\section{REFERENCES}

[1] H. Jiang, W. Zhuang, X. Shen, and Q. Bi, "Quality-of-service provisioning and efficient resource utilization in CDMA cellular communications, ”IEEE J. Sel. Areas Commun., vol. 24, no. 1, pp. 4 15, Jan. 2006.

[2] Y. Chen, N. Yang, and C. Chang, "A utility function-based access selection method for heterogeneous WCDMA and WLAN networks," in Proc. IEEE PIMRC, 2007, pp. 1-5.

[3] Wei Song, Hai Jiang, Weihua Zhuang, and Xuemin (Sherman) Shen, University of Waterloo Resource Management for QoS Support inCellular/WLAN Interworking.

[4] X. Pei, G. Zhu, Q. Wang, D. Qu, and J. Liu, "Economic model-basedradio resource management with QoS guarantees in the CDMA uplink,'Eur. Trans. Telecommun., vol. 20, pp. 1-9, Apr. 2009.

[5] Xuebing Pei, Tao Jiang, Member, IEEE, Daiming Qu, Guangxi Zhu, Senior Member, IEEE, and Jian Liu Radio-Resource Management and Access-Control Mechanism Based on a Novel Economic Model in Heterogeneous Wireless Networks, IEEE TRANSACTIONS ON VEHICULAR TECHNOLOGY, VOL. 59, NO. 6, JULY 2010.

[6] P. Wang, H. Jiang, and W. Zhuang, "Capacity improvement and analysis for voice/data traffic over WLANs,” IEEE Trans. Wireless Commun., vol. 6, no. 4, pp. 1530-1541, Apr. 2007.

[7] M. Buddhikot et al., "Integration of 802.11 and Third-Generation Wireless Data Networks," Proc. IEEE INFOCOM, vol. 1, Apr. 2003, pp. 503-12.

[8] B. Moon and H. Aghvami, "DiffServ Extension for QoS Provisioning in IP Mobility Environments," IEEEWireless Commun., vol. 10, no. 5, Oct. 2003, pp. 38-44. 
[9] Q. Zhang et al., "Efficient Mobility Management for Vertical Handoff between WWAN and WLAN," IEEE Commun. Mag., vol. 41, no. 11, Nov. 2003, pp. 102-08.

[10] T. Klein and S.-J. Han, "Assignment Strategies for Mobile Data Users in Hierarchical Overlay Networks:Performance of Optimal and Adaptive Strategies,” IEEE JSAC, vol. 22, no. 5, June 2004, pp. 849-61.

[11] Safdar Rizvi, Asif Aziz, N.M. Saad, Nasrullah Armi \& Mohd Zuki Yusoff, “Tight Coupling Internetworking Between UMTS and WLAN: Challenges, Design Architectures and Simulation Analysis" International Journal of Computer Networks (IJCN), Volume (3):issue(2) : 2011116.

[12] Salawu Nathaniel, Elizabeth NonyeOnwuka, Abolarinwa Joshua Adegboyega, dauda Umar Suleiman "Networks integration between WirelessLAN and UMTS Networks- A Brief Study" Journal of Emerging Trends in Computing and Information Sciences Vol. 4, No. 5 May 2013.

\section{AUTHOR}

Nilkantha Chakraborty received B.Tech. degree in Electronics and Communication from West Bengal University of Technology and M.Tech. in Communication from VIT University, Tamil Nadu. He is currently working at Cognizant technology solution. His research interests include wireless communication, networking.

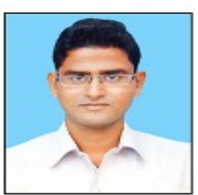

